\title{
Is COVID-19 an Invitation to Reconfigure or Reinvent Pulmonary Rehabilitation Service Delivery?
}

\author{
Shakila Devi Perumal
}

Cardiff University, United Kingdom

\section{Corresponding Author*}

Shakila Devi Perumal

School of Healthcare Sciences, Cardiff University, United Kingdom

E-mail: perumalsd@cardiff.ac.uk

Copyright: 2020 Shakila Devi Perumal. This is an open-access article distributed under the terms of the Creative Commons Attribution License, which permits unrestricted use, distribution, and reproduction in any medium, provided the original author and source are credited.

Received 11 December, 2020; Accepted 15 December, 2020; Published 25 December, 2020

\section{Abstract}

The Outbreak of COVID-19 pandemic demanded a swift reconfiguration of healthcare delivery to mitigate the associated risk and case-fatality. Patients with chronic respiratory disease (CRD) were at higher risk with residual "multiorgan" manifestations of COVID -19. Post-COVID rehabilitation is on high demand to alleviate symptoms and reduce mortality in patients with chronic respiratory disease (CRD). Pulmonary rehabilitation is endorsed as an integrative management, an essential component of respiratory medicine for CRD. However, COVID -19 pandemic has sparked many controversies and challenges on the pulmonary rehabilitation service delivery around the world. This article explores perspectives and action strategies for an agile pulmonary rehabilitation service in the era of COVID-19.

Keywords: COVID-19, Pulmonary rehabilitation, Chronic respiratory disease, Integrated care, Social prescribing

\section{Introduction}

In March 2020, the world health organisation had declared the outbreak of COVID-19 pandemic that exploded overnight unprecedented public health crisis and renewed concerns to economy [1]. As of 6th December 2020, the global cases surpassed 66 million with over 1.5 million deaths [2]. International health organisations had responded with swift healthcare interventions and robust safety measures to mitigate the risks of COVD-19 interventions based on the perceptions it was a "Respiratory Disease". Patients with chronic respiratory disease (CRD) were identified as "high risk" group victimised with persistent symptoms including chronic cough, dyspnoea, fatigue, exercise intolerance, fibrotic changes in lung and myopathies [3]. Social isolation and distancing as a measure of risk stratification has posed challenges of "Fear and loneliness" harming mental wellbeing of patients [4]. Recent growing evidence confirms, COVID-19 is not just respiratory disease; it is multicomponent disease affecting heart muscle, respiratory systems, peripheral nervous system, brain, gastrointestinal and musculoskeletal system [5]. At present, there is limited evidence on the sequelae of illness post- COVID -19 and its management [6]. The uncertain trajectory of COVID imposes severe concern and requires integrative mitigation of healthcare interventions at primary care centre $[7,8]$.

Pulmonary Rehabilitation (PR) is endorsed as a "Gold Standard" integrative respiratory medicine in the management of CRD. International respiratory societies defined PR, as a comprehensive multi-component program that includes patient assessment, patient-tailored therapies of exercise, education, and psycho-social intervention designed for CRD patients to manage their condition beyond the duration of the program [9]. As per definitions, PR is more than "just an exercise program" or "Chest Physiotherapy" and warrants inclusion of multidisciplinary and supplementary interventions to improve skills for "effective long-term behaviour change in patients with COPD". Recent policy statement on PR from international respiratory societies has several recommendations to address the theory-practice gap in PR [10]. Yet, widely
PR is under-utilised and global variations persist with regional heterogenicity in local healthcare systems development [11].

\section{Pre-COVID - 19 Pulmonary Rehabilitation in the United Kingdom}

In 2014, the integration of health and social care systems was the central tenet for the NHS five year forward view. In 2019, the NHS long-term plan identified "Respiratory disease" as a national clinical priority and had a vision to establish integrated care systems (ICS) in every part of the country to reduce hospital admissions [12]. The key focus was committed to the expansion of PR services for CRD putting ownership on primary care networks. The NHS Improvement project aimed to increase the number of patients who would benefit from increasing PR's referral rates from $13 \%$ to $60 \%$ by 2023 . Besides, the NHS improvement project also committed to giving patients digital access to their medical records from 2020/21 and the expansion of mental health services with the integration of physical activity by 2023 . Yet, a recent report on ICS in rural areas (2020), confirmed 14 different ICS model across England are not meeting the expected reductions in hospital admissions [13]. Many reasons being identified: 1 . Lack of patient perspectives 2. Low multidisciplinary working partnership at primary care 3. Lack of SMART outcomes measures.

In the same vein, the BTS position statement on Integrated Care Model [14] had identified two significant inconsistencies in PR service delivery: 1. Access and delivery of post- hospitalization PR 2 . Adherence to guidelines and quality standards. Four key actionable steps were recommended for sustainable PR service delivery:

1. Ongoing digital education support for patients attending pulmonary rehabilitation

2. Integrated digital decision support

3. Incentivizing cross-organization working system design

4. Quality assurance of clinical data collection and audit process

To support the action steps, two significant initiatives such as 1 . NHS longTerm Plan, 2. National GP incentivizes program scheme to build PR capacity and promotion.

\section{Reconfigure or Reinvent Pulmonary Rehabilitation in United Kingdom?}

Covid -19 has brought in both unprecedented challenges and unparalle opportunities for innovations in global health care delivery models, including pragmatic pulmonary rehabilitation services [15]. During the dawn of COVID-19, to manage morbidity and mortality toll, national pulmonary rehabilitation services have been suspended, and all staff have redeployed to frontline care resulting in an increased PR waiting list. The patient-centred care and experience, a central precept of NHS long-term plan, is now made mandatory to build a resilient care system that prioritises value, accessibility, satisfaction, and importantly outcomes.

Evidence confirms $80 \%$ of health outcomes are dictated by social determinants of health, including employment status, education, housing, transportation, and dietary habits [16]. COVID -19 has exacerbated these issues and demands an agile and responsive healthcare delivery model. These health imperatives have placed an undeniable demand on telehealth and virtual solutions that can no longer be set aside. Usage of telehealth and virtual care has already risen steeply in the last eight months. Studies confirm remotely supported PR with telecare is non-inferior to conventional centre-based PR $[17,18]$. The swift shift toward digital delivery provides a clean slate for clinical leaders to understand the current clinical condition and experience of their patient population and staff needs for a sustainable health system. Presently, with ambiguous promises on new vaccines, we are entering into new challenges of Post-COVID rehabilitation.

Guidelines for post-COVID rehabilitation emphasise an integrated multidisciplinary approach involving medical doctors, physiotherapists, and psychotherapists $[14,19]$. Many UK centres yet to establish these services and optimal care management remains unclear. Besides, NHS long-term Plan 
has recognised effective use of nature-based social prescribing is likely to improve patient's physical, social and emotional well begin. Inarguably, this provokes general practitioners and allied healthcare professionals to consider holistic rehabilitation prescription comprising physical, psychological, and social domains to benchmark quality care for patients with CRD. Yet, to date, there is no consensus on mode, duration, intensity of rehabilitation and critical healthcare professionals accountable with parity for care delivery to diverse population in this era of COVID pandemic.

Recent BTS update on "Business as Usual" has documented guidance on revamping PR with alternative business solutions (digital) to ensure the best quality of post COVID rehabilitation. Though there was a swift cultural change of virtual connections of video calling for patient consultation, still there were some unknown barriers for a consistent, integrated care model to deliver consistent and agile PR services. Arguably, it is unknown if COVID-19 is a percussor for a cultural change of technology-based solutions for sustainable care amidst the ambiguity of cybersecurity and integrity of health care data.

Present COVID-19 challenges demand an appealing value-based integrated care delivery in primary, secondary and community care; it warrants an indepth look at the physical, mental, behavioural and social needs of patients with CRD. Utilising the lessons learned for past months, will allow clinical leaders to devote time for development and implementation of agile and sustainable health systems. NHS and partner charity organisations should take this opportunity to not only reconfigure but to reinvent a patient-centric, user-friendly, resilient pulmonary rehabilitation delivery model across the care continuum.

Moving forward, reconfiguration of pulmonary rehabilitation services in primary care should include elements of live, personalised care programme, agile staffing models, continual outcome assessment and patient engagement with parity of health community [20]. Integration of artificial intelligence and augmented reality would be an intuitive way of reinventing pulmonary rehabilitation care delivery to target personalised care and patient engagement [21]. Integrated telerehabilitation would be cost-effective on workforce demands for diagnostics and therapeutic interventions to target the right care, at the right time, for the right people.

\section{Actionable points to be considered:}

Based on the recent evidence, the following actions are suggested to reconfigure agile pulmonary rehabilitation services in response to COVID-19 challenges.

1. A comprehensive PR care should be co-produced with patients based on their bio-psychosocial paradigm and treatable traits to tailor disease management, consultation and behavioural coaching.

2. To address the physical and psycho-social domain, it is highly critical to promote initiatives to implement interdisciplinary rehabilitation and encourage patients to attend primary care as first line of contact. A suggestive strategy would be to consider modular programme delivery by integrating mental health and social prescribing services to target the "multifaceted" manifestations of COVID-19 for CRD patients.

3. To ensure sustainable care, each primary care centre should commission a new role "patient care coordinator" who should be working in collaboration with medical doctors, physiotherapists, psychotherapists, dietician, occupational therapist and social prescriber's network.

4. An appropriate nationwide technology-based for telerehabilitation warrants an in-depth look at artificial intelligence and augmented reality to open opportunities to improve quality and efficiency of pulmonary rehabilitation care delivery.

5. Health policies should be focused on practical implementation strategies to strengthen political and clinical leadership. General physicians at every primary care should become a constructive voice to drive change, share good practice, train relevant workforce with core skills and promote innovations in PR integrated care.

6. To address the knowledge gaps in integrated PR care, it is essential to survey the needs of education, telehealth familiarity and rehab system consultation among respiratory care professionals. National Primary Care Services should consider inclusion of pulmonary rehab as a core module in the curriculum of Medical General Practice and Allied health professionals. All key stakeholders in pulmonary rehabilitation require formal continuous professional development process to drive change and innovation.

7. Formal annual survey on workforce needs and training of specialist is imperative to deliver a high standard of quality care. A framework of continuous quality evaluation: transforming data to knowledge, translating knowledge to performance and evaluating performance with data is imperative in the delivery of PR care services to achieve a safe quality of care.
8. It is critical to translate the clinical findings and communicate it with all stakeholders in respiratory care for patient-centric quality care. For this, investments in partnerships between higher education institutions and local health services is crucial to increase the capacity and capability of the workforce through sustained patient -public engagement research interactions.

9. Appropriate risk stratification tools is vital to prevent health inequalities. All CCG's should consider promoting pragmatics randomised trials or regression analysis to understand and establish evidence-based pulmonary rehabilitation for CRD patients.

10. To balance the demand-supply side, strategies driven by shared vision activate a dynamic and collaborative engagement among the patient, family and healthcare provider to promote sustainable outcomes.

These action points could relieve the overall economic burden on the NHS and enable provision of PR integrated care for CRD population in this unprecedented challenge of COVID-19.

\section{Conclusion}

The success of these proposed action points depends on stakeholder's commitment to shared vision, good planning, digital solutions, reliable data, and realistic funding. As recurring challenges of COVID-19 is constant, it is imperative to reinvent a responsive pulmonary rehabilitation delivery model defined by a holistic, interdisciplinary culture of interventions for an equitable level of treatment, care, and support to patients with CRD.

\section{Funding}

This article received no specific grant from any funding agency in the public, commercial, or not-for-profit sectors.

\section{Reference}

1. World Health Organization (WHO). "Multisystem inflammatory syndrome in children and adolescents with COVID-19". Geneva: WHO, (2020). Retrieved from: https://www.who.int/publications-detail/multisysteminflammatorysyndrome-in-children-and-adolescents-with-covid-19.

2. World Health Organization (WHO) Dashboard. (2020). Retrieved from: https:// covid19.who.int/

3. Carfi, A., et al. "Persistent symptoms in patients after acute COVID-19". JAMA 324(6) (2020), 603-605.

4. Wong, S., et al. "Impact of COVID-19 on loneliness, mental health, and health service utilisation: a prospective cohort study of older adults with multimorbidity in primary care". British Journal of General Practice, 70(700), (2020), e817-e824.

5. Docherty, A., et al. "Features of 20133 UK patients in hospital with covid-19 using the ISARIC WHO Clinical Characterisation Protocol: prospective observational cohort study". BMJ, (2020), m1985.

6. Greenhalgh, T., et al. "Management of post-acute covid-19 in primary care". $B M J, 370$ (2020), m3026.

7. Gemelli against COVID-19 Post-Acute care study group. "Post-COVID-19 global health strategies: the need for an interdisciplinary approach". Aging clinical and experimental research, 32(8) (2020), 1613-1620.

8. Tan, W., et al. "Is returning to work during the COVID-19 pandemic stressful? A study on immediate mental health status and psychoneuroimmunity prevention measures of Chinese workforce". Brain, Behavior, and Immunity, 87 (2020), 84-92.

9. Martijn, A.S., et al. "An official American Thoracic Society/ European Respiratory Society statement: key concepts and advances in pulmonary rehabilitation'. Am J Respir Crit Care Med, 188(8) (2013), e13-64.2013.

10. Rochester, C.L., et al. "An Official American Thoracic Society/European Respiratory Society Policy Statement: Enhancing Implementation, Use, and Delivery of Pulmonary Rehabilitation". Am J Respir Crit Care Med, 192 (2015), 1373-1386.

11. Wallaert, B., et al. "Long-term evaluation of home-based pulmonary rehabilitation in patients with COPD". International Journal of Chronic Obstructive Pulmonary Disease, (2015), 2037.

12. NHS long-term plan. Retrieved from: https://www.longtermplan.nhs.uk/ wp-content/uploads/2019/01/nhs-long-term-plan-june-2019.pdf

13. What does integrated care mean for rural communities? (2020). Retrieved from: https://www.rsnonline.org.uk/what-does-integrated-care-mean-forrural-communities. 
14. British Thoracic Society. Guidance on respiratory follow up of patients with a clinico- radiological diagnosis of covid-19 pneumonia. (2020). Retrieved from: www.brit-thoracic.org.uk.

15. Houchen-Wolloff, L., \& Steiner, M.C. "Pulmonary rehabilitation at a time of social distancing: prime time for tele-rehabilitation?" Thorax, 75 (2020), 446447.

16. Abrams, E., \& Szefler, S. "COVID-19 and the impact of social determinants of health". The Lancet Respiratory Medicine, 8(7) (2020), 659-661.

17. Chaplin, E., et al. "Interactive web-based pulmonary rehabilitation programme: a randomised controlled feasibility trial". BMJ Open, 7 (2017), e 013682.
18. Vasilopoulou, M., et al. "Home-based maintenance tele- rehabilitation reduces the risk for acute exacerbations of COPD, hospitalisations and emergency department visits". The European Respiratory Journal, 49(5) (2017), 2016.

19. NHS England. Aftercare needs of inpatients recovering from COVID-19. (2020), Retrieved from: https://www.england.nhs.uk/coronavirus/publication/aftercare-needs-of-inpatients-recovering-from-covid-19/

20. The Marmot Review Strategic Review of Health Inequalities in England Post2010. Fair Society, Healthy Lives. Retrieved from: https://www.parliament.uk/ documents/fair-society-healthy-lives-full-report.pdf.

21. Kamal, J. "Artificial Intelligence Applications in handling the Infectious Diseases". Prim Health Care, 10(5) (2020), 351 